\title{
Attracting and quasi-invariant sets for a class of impulsive stochastic difference equations
}

\author{
Dingshi Li and Shujun Long
}

\author{
* Correspondence: \\ lidingshi2006@163.com \\ Yangtze Center of Mathematics, \\ Sichuan University, Chengdu \\ 610064, China
}

\begin{abstract}
The aim of this article is to study the attracting and quasi-invariant sets for a class of impulsive stochastic difference equations. By establishing a difference inequality, we obtain the attracting and quasi-invariant sets of systems under consideration. An example is given to illustrate the theory.

Keywords: Attracting set, Quasi-invariant set, Impulsive, Stochastic, Difference equations, Difference in-equality, Halanay inequality
\end{abstract}

\section{Introduction}

Difference equations usually appear in the investigation of systems with discrete time or in the numerical solution of systems with continuous time [1]. A lot of difference systems have variable structures subject to stochastic abrupt changes, which may result from abrupt phenomena such as stochastic failures and repairs of the components, changes in the interconnections of subsystems, sudden environment changes, etc. In recent years, the stability investigation of stochastic difference equations has been interesting to many investigators, and various advanced results on this problem have been reported [2-5].

However, besides the stochastic effect, an impulsive effect likewise exists in a wide variety of evolutionary processes in which states are changed abruptly at certain moments of time, involving such fields as medicine and biology, economics, mechanics, electronics and telecommunications. Recently, the asymptotic behaviors of impulsive difference equations have attracted considerable attention. Many interesting results on impulsive effect have been obtained [6-8]. In [9], some stability conditions on impulsive stochastic difference equations are given. As is well known, stability is one of the major problems encountered in applications, and has attracted considerable attention due to its important role in applications. However, under impulsive perturbation, an equilibrium point sometimes does not exist in many physical systems, especially, in non-linear and nonautonomous dynamical systems. Therefore, an interesting subject is to discuss the invariant sets and the attracting sets of impulsive systems. Some significant progress has been made in the techniques and methods of determining the invariant sets and attracting sets for delay difference equations, delay differential equations, and impulsive functional differential equations [10-12]. Unfortunately, the corresponding problems for impulsive stochastic difference equations have not been considered.

\section{SpringerOpen ${ }^{\circ}$}

(C) 2011 Li and Long; licensee Springer. This is an Open Access article distributed under the terms of the Creative Commons Attribution License (http://creativecommons.org/licenses/by/2.0), which permits unrestricted use, distribution, and reproduction in any medium, provided the original work is properly cited. 
Motivated by the above discussion, we here make a first attempt to arrive at results on the invariant sets and attracting sets of impulsive stochastic difference equations.

\section{Model description and preliminaries}

Let $R^{n}$ be the space of $n$-dimensional real column vectors and $R_{+}=[0,+\infty) . N[a, b]\{a$, $a+1, \ldots, b\}$, where $a<b$ and $a, b$ are integral numbers. $C$ denotes the set of all functions $\varphi: N[-h, 0] \rightarrow R^{n}, h$ is a nonnegative integer. For any $\phi \in C$, we define $\|\varphi\|=\sup _{s \in N[-h, 0]}|\varphi(s)| . Z$ denotes the integer set. Let $\{\Omega, P, \Sigma\}$ be a basic probability space, $\Sigma_{i-1} \subset \Sigma_{i} \subset \Sigma, i \in Z$ be a sequence of $\Sigma$-algebras $E$ be the mathematical expectation, $\xi_{0}, \xi_{1}, \ldots$ be a sequence of mutually independent random variables, $\xi_{i} \in R$, $\xi_{i}$ be $\Sigma_{i^{-}}$ adapted and independent on. $\Sigma_{i-1}, E \xi_{i}=0, E \xi_{i}^{2}=1, i \in Z$. Let $C_{\Omega}$ denote the family of $C$-valued random variables on $\{\Omega, P, \Sigma\}$.

In this article, we mainly consider the following impulsive stochastic difference equations

$$
\left\{\begin{array}{l}
x(i+1)=F(i, x(i-h), \ldots, x(i))+G(i, x(i-h), \ldots, x(i)) \xi_{i+1}, \quad i \neq i_{k}, i \in Z, \\
x(i+1)=H_{i}(x(i)), \quad i=i_{k}
\end{array}\right.
$$

with initial condition

$$
x(i)=\varphi(i), \quad i \in N[-h, 0],
$$

where $F, G: Z \times R^{h+1} \rightarrow R, H_{i}: R \rightarrow R . \phi(i) \in C_{\Omega}$. The fixed moments of time $i_{k} \in$ $Z$, and satisfy $0<i_{1}<i_{2}<\ldots, \lim _{k \rightarrow \infty} i_{k}=\infty . x_{i}$ is an element of $C_{\Omega}$ defined by $x_{i}=x(i$ $+s), s \in N[-h, 0]$.

Throughout this article, we assume that for any $\phi(i) \in C_{\Omega}$, there exists at least one solution of (1), which is denoted by $x(i, 0, \phi)$ or $x_{i}(0, \phi)$ (simply $x(i)$ and $x_{i}$ if no confusion should occur).

Definition 2.1. The set $S \subset C_{\Omega}$ is called a quasi-invariant set of (1), if there exists a constant $k$ such that for any initial value $\phi \in S$, the solution $k x_{i}(0, \phi) \in S, i \in Z$. Especially, if $k=1, \mathrm{~S}$ is called a invariant set.

Dedinition 2.2. The set $S \subset C_{\Omega}$ is called a global attracting set of (1), if for any initial value $\phi \in C_{\Omega}$, the solution $x_{i}(0, \phi)$ satisfies

$$
\operatorname{dist}\left(x_{i}, S\right) \rightarrow 0, \quad \text { as } \quad i \rightarrow \infty,
$$

where

$$
\operatorname{dist}(\varphi, S)=\inf _{\psi \in S} \rho(\varphi(s), \psi(s)) \quad \text { for } \quad \varphi \in C_{\Omega}
$$

where $\rho(\cdot, \cdot)$ is any distance in $C_{\Omega}$.

Definition 2.3. The zero solution of Equation (1) is called mean square exponential stable if there are positive constants $\lambda$ and $M$ such that for any initial condition $\phi \in$ $C_{\Omega}$,

$$
E x^{2}(i) \leq M E\|\varphi\|^{2} e^{-\lambda i}, \quad i \in Z
$$

Here $\lambda$ is called the exponential convergence rate. Of course, conditions are needed to ensure that the zero function is a solution of (1). 
Based on discrete Halanay inequality [13] and its extension [9], we develop the following difference in-equality with the impulsive initial condition.

Lemma 2.1. Suppose $c_{j}(i) \in R_{+}, i \in Z, j \in N[0, h], \sup _{i \in Z}\left\{\sum_{j=0}^{h} c_{j}(i)\right\}=\eta<1$ and $b>0$.

Let $u(i)$ be a sequence of real numbers satisfying the following difference inequality:

$$
u(i+1) \leq \sum_{j=0}^{h} c_{j}(i) u(i-j)+b, \quad i \geq i^{\prime}, \quad i \in Z .
$$

(a) Then

$$
u(i) \leq d e^{-\lambda i}+(1-\eta)^{-1} b, \quad i \geq i^{\prime}, \quad i \in Z,
$$

provided that the initial condition satisfies

$$
u(i) \leq d e^{-\lambda i}+(1-\eta)^{-1} b, \quad i \in N\left[i^{\prime}-h, i^{\prime}\right],
$$

where $i^{\prime} \in Z, d \in R_{+}$and $\lambda$ satisfies

$$
0<\lambda \leq \frac{1}{h+1} \ln \frac{1}{\eta}
$$

(b) Then

$$
u(i) \leq \gamma(1-\eta)^{-1} b, \quad i \geq i^{\prime}, \quad i \in Z,
$$

provided the initial condition

$$
u(i) \leq \gamma(1-\eta)^{-1} b, \quad i \in N\left[i^{\prime}-h, i^{\prime}\right],
$$

where $i^{\prime} \in Z$ and $\gamma \geq 1$.

Proof. (a) Since $\eta<1$, there exists a constant $\lambda$ satisfying the inequality (5). Then,

$$
e^{\lambda(h+1)} \eta \leq 1
$$

If (3) is not true, then there must be a positive integral number $i^{*} \geq i$ ' such that

$$
u\left(i^{*}+1\right)>d e^{-\lambda\left(i^{*}+1\right)}+(1-\eta)^{-1} b \quad \text { and } \quad u(i) \leq d e^{-\lambda i}+(1-\eta)^{-1} b, \quad i \in N\left[i^{\prime}-h, i^{*}\right] .
$$

By (2), (8), and (9), we have

$$
\begin{aligned}
u\left(i^{*}+1\right) & \leq \sum_{j=0}^{h} c_{j}\left(i^{*}\right) u\left(i^{*}-j\right)+b \\
& \leq \sum_{j=0}^{h} c_{j}\left(i^{*}\right)\left[d e^{-\lambda\left(i^{*}-j\right)}+(1-\eta)^{-1} b\right]+b \\
& \leq e^{\lambda(h+1)} \eta d e^{-\lambda\left(i^{*}+1\right)}+\eta(1-\eta)^{-1} b+b \\
& =e^{\lambda(h+1)} \eta d e^{-\lambda\left(i^{*}+1\right)}+(1-\eta)^{-1} b \\
& \leq d e^{-\lambda\left(i^{*}+1\right)}+(1-\eta)^{-1} b,
\end{aligned}
$$


which contradicts the first inequality of (9). So (3) holds. The proof of part (a) is complete. (b) If (6) is not true, then there must be a positive integral number $i^{*} \geq i$ ' such that

$$
u\left(i^{*}+1\right)>\gamma(1-\eta)^{-1} b \quad \text { and } \quad u(i) \leq \gamma(1-\eta)^{-1} b, \quad i \in N\left[i^{\prime}-h, i^{*}\right] .
$$

By (2), (10), we have

$$
\begin{aligned}
u\left(i^{*}+1\right) & \leq \sum_{j=0}^{h} c_{j}\left(i^{*}\right) u\left(i^{*}-j\right)+b \\
& \leq \gamma \eta(1-\eta)^{-1} b+b \\
& \leq \gamma\left(\eta(1-\eta)^{-1} b+b\right) \\
& =\gamma(1-\eta)^{-1} b,
\end{aligned}
$$

which contradicts the first inequality of (10). So (6) holds. The proof of part (b) is complete.

\section{Main results}

To establish the main results of system (1), we will employ the following assumptions.

$\left(\mathrm{A}_{1}\right)$ For any $i \in Z$, there exist positive constants $a_{j}(i), b_{j}(i), J_{1}$ and $J_{2}$ such that

$$
\begin{aligned}
& |F(i, x(i-h), \ldots, x(i))| \leq \sum_{j=0}^{h} a_{j}(i)|x(i-j)|+J_{1}, \\
& |G(i, x(i-h), \ldots, x(i))| \leq \sum_{j=0}^{h} b_{j}(i)|x(i-j)|+J_{2} .
\end{aligned}
$$

$\left(\mathrm{A}_{2}\right) \sup _{i \in Z} 2\left\{a^{2}(i)+b^{2}(i)\right\}=\mu<1$, where $a(i)=\sum_{j=0}^{h} a_{j}(i)$ and $b(i)=\sum_{j=0}^{h} b_{j}(i)$.

$\left(\mathrm{A}_{3}\right)$ There exist constants $d_{k} \geq 1$ such that

$$
\left|H_{i_{k}}\left(x\left(i_{k}\right)\right)\right| \leq d_{k}\left|x\left(i_{k}\right)\right|, \quad k=1,2, \ldots .
$$

(A $\left.\mathrm{A}_{4}\right)$ There exists constant $\alpha \geq 0$ such that

$$
\frac{2 \ln d_{k}}{i_{k}-i_{k-1}} \leq \alpha^{*}<\lambda^{*}, \quad k=1,2, \ldots
$$

where $i_{0}=0$ and $\lambda^{*}$ satisfies

$$
0<\lambda^{*}=\frac{1}{h+1} \ln \frac{1}{\mu}
$$

and

$$
\sigma=2 \sum_{k=1}^{\infty} \ln d_{k}<\infty, \quad k=1,2, \ldots
$$

$\left(\mathrm{A}_{5}\right)$ There exist nonnegative constants $d_{k} \leq 1$ such that

$$
\left|H_{i_{k}}\left(x\left(i_{k}\right)\right)\right| \leq d_{k}\left|x\left(i_{k}\right)\right| .
$$


$\left(\mathrm{A}_{6}\right)$ For any $i \in Z$, there exist positive constants $a_{j}(i)$ and $b_{j}(i)$ such that

$$
\begin{aligned}
& |F(i, x(i-h), \ldots, x(i))| \leq \sum_{j=0}^{h} a_{j}(i)|x(i-j)|, \\
& |G(i, x(i-h), \ldots, x(i))| \leq \sum_{j=0}^{h} b_{j}(i)|x(i-j)| .
\end{aligned}
$$

$\left(\mathrm{A}_{7}\right) \sup _{i \in Z}\left\{a^{2}(i)+b^{2}(i)\right\}=\mu<1$, where $a(i)=\sum_{j=0}^{h} a_{j}(i)$ and $b(i)=\sum_{j=0}^{h} b_{j}(i)$.

Theorem 3.1. If $\left(\mathrm{A}_{1}\right)-\left(\mathrm{A}_{4}\right)$ hold, then $S=\left\{\varphi \in C_{\Omega}|E| \mid \varphi \|^{2} \leq e^{\sigma}(1-\mu)^{-1} J\right\}$ is a global attracting set of $(1)$, where $J=2\left(J_{1}^{2}+J_{2}^{2}\right)$.

Proof. From (1), Condition $\left(\mathrm{A}_{1}\right),(a+b)^{2} \leq 2\left(a^{2}+b^{2}\right)$ and the Hölder inequality, we have

$$
\begin{aligned}
E x^{2}(i+1) & =E F^{2}(i, x(i-h), \ldots, x(i))+E G^{2}(i, x(i-h), \ldots, x(i)) \\
& \leq E\left(\sum_{j=0}^{h} a_{j}(i)|x(i-j)|+J_{1}\right)^{2}+E\left(\sum_{j=0}^{h} b_{j}(i)|x(i-j)|+J_{2}\right)^{2} \\
& \leq 2 E\left(\sum_{j=0}^{h} a_{j}(i)|x(i-j)|\right)^{2}+2 E\left(\sum_{j=0}^{h} b_{j}(i)|x(i-j)|\right)^{2}+2\left(J_{1}^{2}+J_{2}^{2}\right) \\
& \leq 2 \sum_{j=0}^{h} a_{j}(i) \sum_{j=0}^{h} a_{j}(i) E|x(i-j)|^{2}+2 \sum_{j=0}^{h} b_{j}(i) \sum_{j=0}^{h} b_{j}(i) E|x(i-j)|^{2}+J \\
& =2 \sum_{j=0}^{h}\left[a(i) a_{j}(i)+b(i) b_{j}(i)\right] E x^{2}(i-j)+J, i \neq i_{k}, k=1,2, \ldots
\end{aligned}
$$

From condition $\left(\mathrm{A}_{2}\right)$, we obtain

$$
\sup _{i \in Z} 2 \sum_{j=0}^{h}\left[a(i) a_{j}(i)+b(i) b_{j}(i)\right]=\sup _{i \in Z} 2\left\{a^{2}(i)+b^{2}(i)\right\}=\mu<1 .
$$

For the initial conditions $x(s)=\phi(s), s \in N[-h, 0]$, where $\phi \in C_{\Omega}$, we have a positive constant $K$ such that

$$
E x^{2}(i) \leq K e^{-\lambda^{*} i}+(1-\mu)^{-1} J, \quad i \in N[-h, 0]
$$

Then, all the conditions of the part (a) of Lemma 2.1 are satisfied by (11)-(13). So, we can obtain

$$
E x^{2}(i) \leq K e^{-\lambda^{*} i}+(1-\mu)^{-1} J, \quad i \in N\left[0, i_{1}\right] .
$$

Suppose for all $q=1,2, \ldots, k$, the inequalities

$$
E x^{2}(i) \leq d_{0}^{2} d_{1}^{2} \cdots d_{q-1}^{2} K e^{-\lambda^{*} i}+d_{0}^{2} d_{1}^{2} \cdots d_{q-1}^{2}(1-\mu)^{-1} J, \quad i \in N\left[i_{q-1}, i_{q}\right],
$$

hold, where $d_{0}=1$ and $i_{0}=0$. Then from condition $\left(\mathrm{A}_{3}\right)$ and (14), we have

$$
\begin{aligned}
E x^{2}\left(i_{k}+1\right) & =E\left|H_{i_{k}}\left(x\left(i_{k}\right)\right)\right|^{2} \\
& \leq \mid d_{k}^{2} E x^{2}\left(i_{k}\right) \\
& \leq d_{0}^{2} d_{1}^{2} \cdots d_{k-1}^{2} d_{k}^{2} K e^{-\lambda^{*} i}+d_{0}^{2} d_{1}^{2} \cdots d_{k-1}^{2} d_{k}^{2}(1-\mu)^{-1} J .
\end{aligned}
$$


This, together with (14) and $d_{k} \geq 1, k=1,2, \ldots$, leads to

$$
E x^{2}(i) \leq d_{0}^{2} d_{1}^{2} \cdots d_{k-1}^{2} d_{k}^{2} K e^{-\lambda^{*} i}+d_{0}^{2} d_{1}^{2} \cdots d_{k-1}^{2} d_{k}^{2}(1-\mu)^{-1} J, \quad i \in N\left[i_{k}+1-h, i_{k}+1\right] .
$$

It follows from (11), (12), (15), and the part (a) of Lemma 2.1 that

$$
E x^{2}(i) \leq d_{0}^{2} d_{1}^{2} \cdots d_{k-1}^{2} d_{k}^{2} K e^{-\lambda^{*} i}+d_{0}^{2} d_{1}^{2} \cdots d_{k-1}^{2} d_{k}^{2}(1-\mu)^{-1} J, \quad i \in N\left[i_{k}+1, i_{k+1}\right],
$$

yielding, together with (14) that

$$
E x^{2}(i) \leq d_{0}^{2} d_{1}^{2} \cdots d_{k-1}^{2} d_{k}^{2} K e^{-\lambda^{*} i}+d_{0}^{2} d_{1}^{2} \cdots d_{k-1}^{2} d_{k}^{2}(1-\mu)^{-1} J, \quad i \in N\left[i_{k}, i_{k}+1\right] .
$$

By mathematical induction, we can conclude that

$$
E x^{2}(i) \leq d_{0}^{2} d_{1}^{2} \cdots d_{k-1}^{2} K e^{-\lambda^{*} i}+d_{0}^{2} d_{1}^{2} \cdots d_{k-1}^{2}(1-\mu)^{-1} J, \quad i \in N\left[i_{k-1}, i_{k}\right], k=1,2, \ldots .
$$

Noticing that $d_{k}^{2} \leq e^{\alpha^{*}\left(i_{k}-i_{k-1}\right)}$ and $e^{\sigma}=\prod_{k=1}^{\infty} d_{k}^{2}<\infty$, by condition $\left(\mathrm{A}_{4}\right)$, we can use (16) to conclude that

$$
\begin{aligned}
E x^{2}(i) & \leq e^{\alpha^{*}\left(i_{1}-i_{0}\right)} \cdots e^{\alpha^{*}\left(i_{k-1}-i_{k-2}\right)} K e^{-\lambda^{*} i}+d_{0}^{2} d_{1}^{2} \cdots d_{k-1}^{2}(1-\mu)^{-1} J \\
& \leq \kappa e^{\alpha^{*} i} e^{-\lambda^{*} i}+e^{\sigma}(1-\mu)^{-1} J \\
& =\kappa e^{-\left(\lambda^{*}-\alpha^{*}\right) i}+e^{\sigma}(1-\mu)^{-1} J, \quad i \in N\left[i_{k-1}, i_{k}\right], k=1,2, \ldots
\end{aligned}
$$

This implies that the conclusion holds and the proof is complete.

Theorem 3.2. If $\left(\mathrm{A}_{1}\right)-\left(\mathrm{A}_{4}\right)$ hold, then $S=\left\{\left.\varphi \in C_{\Omega}|E||\varphi|\right|^{2} \leq \gamma(1-\mu)^{-1} J, \gamma \geq 1\right\}$ is a quasi-invariant set of (1).

Proof. For the initial conditions $x(s)=\phi(s), s \in N[-\mathrm{h}, 0]$, where $\phi \in S$ we have

$$
E x^{2}(i) \leq \gamma(1-\mu)^{-1} J, \quad i \in N[-h, 0] .
$$

By (17) and the part (b) of Lemma 2.1, we have

$$
E x^{2}(i) \leq \gamma(1-\mu)^{-1} J, \quad i \in N\left[0, i_{1}\right]
$$

Suppose for all $q=1,2, \ldots, k$, the inequalities

$$
E x^{2}(i) \leq d_{0}^{2} d_{1}^{2} \cdots d_{q-1}^{2} \gamma(1-\mu)^{-1} J, \quad i \in N\left[i_{q-1}, i_{q}\right],
$$

hold, where $d_{0}=1$ and $i_{0}=0$. Then from condition $\left(\mathrm{A}_{3}\right)$ and (18), we have

$$
\begin{aligned}
E x^{2}\left(i_{k}+1\right) & =E\left|H_{i_{k}}\left(x\left(i_{k}\right)\right)\right|^{2} \\
& \leq d_{k}^{2} E x^{2}\left(i_{k}\right) \\
& \leq d_{0}^{2} d_{1}^{2} \cdots d_{k-1}^{2} d_{k}^{2} \gamma(1-\mu)^{-1} J
\end{aligned}
$$

This, together with (18) and $d_{k} \geq 1, k=1,2, \ldots$, leads to

$$
E x^{2}(i) \leq d_{0}^{2} d_{1}^{2} \cdots d_{k-1}^{2} d_{k}^{2} \gamma(1-\mu)^{-1} J, \quad i \in N\left[i_{k}+1-h, i_{k}+1\right] .
$$

It follows from (19) and the part (b) of Lemma 2.1 that

$$
E x^{2}(i) \leq d_{0}^{2} d_{1}^{2} \cdots d_{k-1}^{2} d_{k}^{2} \gamma(1-\mu)^{-1} J, \quad i \in N\left[i_{k}+1, i_{k+1}\right],
$$

yielding, together with (18), that

$$
E x^{2}(i) \leq d_{0}^{2} d_{1}^{2} \cdots d_{k-1}^{2} d_{k}^{2} \gamma(1-\mu)^{-1} J, \quad i \in N\left[i_{k}, i_{k+1}\right] .
$$

By mathematical induction, we can conclude that

$$
E x^{2}(i) \leq d_{0}^{2} d_{1}^{2} \cdots d_{k-1}^{2} \gamma(1-\mu)^{-1} J, \quad i \in N\left[i_{k-1}, i_{k}\right], k=1,2, \ldots
$$


Noticing that $e^{\sigma}=\prod_{k=1}^{\infty} d_{k}^{2}<\infty$,by condition $\left(\mathrm{A}_{4}\right)$, we can use (20) to conclude that

$$
\begin{aligned}
E x^{2}(i) & \leq d_{0}^{2} d_{1}^{2} \cdots d_{k-1}^{2} \gamma(1-\mu)^{-1} J \\
& \leq e^{\sigma} \gamma(1-\mu)^{-1} J, \quad i \in N\left[i_{k-1}, i_{k}\right], k=1,2, \ldots
\end{aligned}
$$

This implies that the conclusion holds and the proof is complete.

Theorem 3.3. If $\left(\mathrm{A}_{1}\right)-\left(\mathrm{A}_{2}\right)$ and $\left(\mathrm{A}_{5}\right)$ hold, then $S=\left\{\varphi \in C_{\Omega}|E||\varphi|^{2} \leq \gamma(1-\mu)^{-1} \beta\right.$ is a invariant set and also a global attracting set of (1).

Proof. Since $d_{k} \leq 1$, a direct calculation shows that $\alpha^{*}=0$ and $\sigma=0$ in Theorems 3.1 and 3.2. It follows from Theorem 3.1 the set $S$ is a global attracting set of (1). It follows from Theorem 3.2 the set $S$ is a invariant set of (1).

If $H_{i_{k}}\left(x\left(i_{k}\right)\right) \equiv x\left(i_{k}\right), k=1,2, \ldots$, the system (1) reduce to the following system without impulses

$$
x(i+1)=F(i, x(i-h), \ldots, x(i))+G(i, x(i-h), \ldots, x(i)) \xi_{i+1}, \quad i \in Z,
$$

with initial condition

$$
x(i)=\varphi(i), \quad i \in N[-h, 0] .
$$

By Theorem 3.3, we can obtain the following result.

Corollary 3.1. If ( $\left.\mathrm{A}_{1}\right)$ and (A2) hold, then $S=\left\{\varphi \in C_{\Omega}|E| \mid \varphi \|^{2} \leq(1-\mu)^{-1}\right\}$ is a invariant set and also a global attracting set of (21).

We easily observe $x(i)=0$ is a solution of (1) from $\left(\mathrm{A}_{3}\right)$ and $\left(\mathrm{A}_{6}\right)$. In the following, we give the attractivity of the zero solution and the proof is similar to that of Theorem 3.1.

Theorem 3.4. If $\left(A_{3}\right),\left(A_{4}\right),\left(A_{6}\right)$, and $\left(A_{7}\right)$ hold, then the zero solution of Equation (1) is mean square exponential stable and the exponential convergence rate is equal to $\lambda^{*}-\alpha^{*}$.

\section{Example}

In this section, we shall discuss an example in order to illustrate the effectiveness of our results. Example 4.1. Consider the following impulsive stochastic difference equation:

$$
\left\{\begin{array}{l}
x(i+1)=\frac{1}{4} \sin (x(i))-\frac{1}{3} x(i-1)+1+\frac{1}{2} x(i) \xi_{i+1}, \quad i \neq i_{k \prime}, \quad i \in Z, \\
x\left(i_{k}+1\right)=e^{1 / 25^{k}} x\left(i_{k}\right), \quad i=i_{k \prime}
\end{array}\right.
$$

where $i_{k}=i_{k-1}+5 k$. Thus,

$$
\begin{aligned}
& h=1, \quad F(i, x(i-h), \ldots, x(i))=\frac{1}{4} \sin (x(i))-\frac{1}{3} x(i-1)+1, \\
& G(i, x(i-h), \ldots, x(i))=\frac{1}{2} x(i), \quad H_{i_{k}}\left(x\left(i_{k}\right)\right)=e^{1 / 25^{k}} x\left(i_{k}\right),
\end{aligned}
$$

yielding

$$
\begin{aligned}
|F(i, x(i-h), \ldots, x(i))| & \leq \frac{1}{4}|x(i)|+\frac{1}{3}|x(i-1)|+1 \\
|G(i, x(i-h), \ldots, x(i))| & \leq \frac{1}{2}|x(i)|, \quad\left|H_{i_{k}}\left(x\left(i_{k}\right)\right)\right|=e^{1 / 25^{k}}\left|x\left(i_{k}\right)\right| .
\end{aligned}
$$


So, the parameters of conditions $\left(\mathrm{A}_{1}\right),\left(\mathrm{A}_{2}\right)$, and $\left(\mathrm{A}_{3}\right)$ are as follows:

$$
\begin{aligned}
& a_{0}(i)=\frac{1}{4}, \quad a_{1}(i)=\frac{1}{3}, \quad b_{0}(i)=\frac{1}{3}, \quad b_{1}(i)=0, \quad J_{1}=1, J_{2}=0, \quad i \in Z, \\
& \mu=\frac{85}{144}<1, \quad d_{k}=e^{1 / 25^{k}}>1, \quad k=1,2, \ldots
\end{aligned}
$$

Since $\frac{2 \ln d_{k}}{i_{k}-i_{k-1}}=\frac{2 \ln e^{1 / 25^{k}}}{5 k}=\frac{2}{25^{k} \times 5 k} \leq 0.016$, we can get $\alpha^{*}=0.016$ and $\lambda^{*}=0.52 \ln \frac{85}{144}$. Moreover, $\sigma=2 \sum_{k=1}^{\infty} \ln d_{k}=2 \sum_{k=1}^{\infty} \ln e^{1 / 25^{k}}=\frac{1}{12}$. Then the condition $\left(\mathrm{A}_{4}\right)$ is satisfied. So, by Theorem 3.1, we can get that

$$
S=\left\{\phi \in C_{\Omega}|E| \mid \phi \|^{2} \leq e^{\sigma}(1-\mu)^{-1} J=\frac{144}{59} e^{\frac{1}{12}}\right\}
$$

is a global attracting set of (22). By Theorem 3.2, we can get that $\mathrm{S}$ is a quasi-invariant set of (22).

\section{Conclusion}

The aim of this article is to study the attracting and quasi-invariant sets for a class of impulsive stochastic difference equations. By establishing a difference inequality, we obtain the attracting and quasi-invariant sets of systems under consideration. As pointed out by the reviewer, when $F$ and $G$ do not depend on $i$, the solutions of (1) are time-homogeneous Markovian in character except that there is an impulse at predetermined times $i_{1}, i_{2}, i_{3} \ldots$. For time-homogeneous Markov chains there is a wellestablished stability theory most eloquently summarized by Meyn and Tweedie [14]. We will explore the relationship between our work and the established theory of stochastic stability for Markov chains in the next article.

\section{Acknowledgements}

The work is supported by National Natural Science Foundation of China under Grant 10971147 . The authors would like to thank the referees for their detailed comments and valuable suggestions which considerably improved the presentation of the paper.

\section{Authors' contributions}

$\mathrm{DL}$ carried out the main proof of the theorems in this paper. SL carried out the expample. All authors read and approve the final manuscript.

\section{Competing interests}

The authors declare that they have no competing interests.

Received: 25 December 2010 Accepted: 17 May 2011 Published: 17 May 2011

\section{References}

1. Kolmanovskii VB, Shaikhet LE: Control of Systems with Aftereffect. In Translations of Mathematical Monographs. Volume 157. American Mathematical Society, Providence, Rl; 1996.

2. Kuchkina N, Shaikhet L: Optimal control of Volterra type stochastic difference equations. Comput Math App/ 1998, 36(10-12):251-259.

3. Taniguchi T: Stability theorems of stochastic difference equations. J Math Anal App/ 1990, 147(1):81-96.

4. Ma F, Caughey TK: Mean stability of stochastic difference systems. Int J Non-Linear Mech 1982, 17(2):69-84

5. Ahmadi G: On the mean square stability of linear difference equations. App/ Math Comput 1979, 5(3):233-241.

6. He ZM, Zhang XM: Monotone iterative technique for first order impulsive difference equations with periodic boundary conditions. Appl Math Comput 2004, 156:605-620.

7. Zhang QQ: On a linear delay difference equation with impulses. Ann Diff Equ 2002, 18(2):197-204.

8. Zhu W, Xu DY, Yang ZC: Global exponential stability of impulsive delay difference equation. Appl Math Comput 2006, 181:65-72 
9. Yang ZG, Xu DY: Mean square exponential stability of impulsive stochastic difference equations. Appl Math Lett 2007, 20:938-945.

10. Xu DY: Asymptotic behavior of nonlinear difference equations with delays. Comput Math Appl 2001, 42:393-398

11. Seifert G: Positively invariant closed sets for systems of delay differential equations. J Diff Equ 1976, 22:292-304.

12. Xu DY, Yang ZC: Attracting and invariant sets for a class of impulsive functional differential equations. J Math Anal Appl 2007, 329:1036-1044.

13. Liz E, Ferreiro JB: A note on the global stability of generalized difference equations. Appl Math Lett 2002, 15:655-659.

14. Meyn SP, Tweedie R: Markov Chains and Stochastic Stability. Springer-Verlag, London; 1993.

doi:10.1186/1687-1847-2011-3

Cite this article as: $L i$ and Long: Attracting and quasi-invariant sets for a class of impulsive stochastic difference equations. Advances in Difference Equations 2011 2011:3.

\section{Submit your manuscript to a SpringerOpen ${ }^{\circ}$} journal and benefit from:

- Convenient online submission

- Rigorous peer review

- Immediate publication on acceptance

- Open access: articles freely available online

- High visibility within the field

- Retaining the copyright to your article

Submit your next manuscript at $\boldsymbol{s p r i n g e r o p e n . c o m ~}$ 